\title{
Timing of administration of indocyanine green for fluorescence-guided surgery in pancreatic cancer: response to Shirakawa et al
}

Peter L. Labib (1)

Keywords: Pancreatic cancer, Fluorescence guided surgery, Indocyanine green, ICG, Staging laparoscopy, Pancreaticoduodenectomy, Metastasis

\section{Main text}

Dear editors,

I read with interest the recent publication of a study protocol for using near-infrared fluorescence imaging with indocyanine green (ICG) during staging laparoscopy for pancreatic cancer (Shirakawa et al) [1]. As the authors state, staging laparoscopy reduces the probability of finding metastatic disease on subsequent laparotomy, but the risk is still 18\% [2]. To prevent unnecessary laparotomies on patients with unresectable pancreatic cancer, trials investigating adjuncts for staging laparoscopy to improve detection of metastatic disease are warranted and welcome.

Previous results on the use of ICG in pancreatic cancer for fluorescence-guided surgery have been conflicting based on the anatomical site of pancreatic cancer being assessed and the timing of ICG administration. ICG is rapidly taken up by hepatocytes and is excreted into the biliary system without re-entering the hepatobiliary circulation [3]. Its ability to highlight liver metastases relies on clearance of ICG from the normal liver parenchyma whilst having impaired clearance from hepatic tumour sites, providing a meaningful tumour-to-

This comment refers to the article available at https://doi.org/10.1186/ s12893-019-0635-0.

Correspondence: peter.labib@nhs.net

Department of hepatopancreaticobiliary surgery, Derriford Hospital, Derriford Road, Plymouth PL6 8DH, UK background ratio (TBR) detectable to the surgeon. To allow time for ICG clearance, ICG needs to be administered at least $24 \mathrm{~h}$ prior to staging laparoscopy (and potentially longer if the patient has any evidence of biliary obstruction). Near-infrared imaging of the primary tumour using ICG has also shown varying results due to timing of administration. Newton and colleagues published two studies $(n=4$ and $n=12)$ demonstrating that after preoperative (24. h) ICG administration, TBRs of 4.64.9 could be achieved in the primary tumour site $[4,5]$. However, a study by Hutteman et al. failed to generate meaningful TBRs with intraoperative ICG administration due to physiological uptake in the normal pancreas (likely related to its high blood supply compared to the tumour mass). Regarding peritoneal deposits, studies suggest that ICG clearance is quite rapid. The paper referenced by the authors by Liberale and colleagues describes the administration of intraoperative ICG for the identification of colorectal peritoneal metastases [6]. Their protocol was changed from preoperative ( $24 \mathrm{~h}$ ) ICG administration to intraoperative ICG administration after the first patient as no near-infrared signal was detected at laparoscopy $24 \mathrm{~h}$ post-administration. Supporting this is their exclusion from further analysis of nodules resected from patients more than six hours post-ICG injection as the fluorescent signal had dissipated. Handgraaf and colleagues published a prospective study combining laparoscopic ultrasound and preoperative (24-48 h) ICG administration in staging 
laparoscopy for pancreatic $\operatorname{cancer}(n=25)$ [7]. Four patients had metastatic disease, two of which were identified by ICG. In the two false negatives, one hepatic metastasis was identified deep to the liver surface by ultrasound and the fourth patient had peritoneal deposits that did not fluoresce.

The problem with the current study protocol is that the primary outcome is trying to simultaneously identify two types of metastatic deposit (hepatic and peritoneal) that have different ideal times for ICG administration. The current protocol for administering ICG one day before staging laparoscopy is ideally suited to the detection of hepatic metastases and pancreatic cancer at the primary site. However, it is unlikely to detect peritoneal deposits as the ICG will have dissipated by this time point. Intraoperative ICG is more likely to detect peritoneal deposits based on the study by Liberale, but this will result in high physiological fluorescence from the liver making identification of small hepatic metastases impossible. One suggested solution is to administer a first bolus of ICG $24 \mathrm{~h}$ preoperatively to assess for hepatic metastases after white light imaging as per the current protocol, but to then administer a second bolus of ICG intraoperatively to detect peritoneal metastases. This amendment may identify more metastatic disease than the current protocol, which may ultimately have a significant effect on the study's primary and secondary outcomes.

I wish the authors good luck in their study and look forward to seeing the results in due course.

\section{Abbreviations}

ICG: Indocyanine green; TBR: Tumour-to-background ratio

\section{Acknowledgements}

Not applicable.

\section{Author's contributions}

$\mathrm{PL}$ was responsible for the conception and writing of this manuscript. All

author(s) read and approved the final manuscript.

\section{Authors' information}

$\mathrm{PL}$ is a surgical trainee in the United Kingdom who is currently undertaking a $\mathrm{PhD}$ at University College London focusing on biophotonic diagnostics and image-guided surgery in pancreatic cancer

\section{Funding}

Not applicable.

Availability of data and materials

Not applicable.

Ethics approval and consent to participate

Not applicable.

Consent for publication

Not applicable.

\section{Competing interests}

The author declares that they have no competing interests.
Received: 4 December 2019 Accepted: 23 September 2020

Published online: 07 October 2020

References

1. Shirakawa S, Toyama H, Kido M, Fukumoto T. A prospective single-center protocol for using near-infrared fluorescence imaging with indocyanine green during staging laparoscopy to detect small metastasis from pancreatic cancer. BMC Surg. 2019;19:165.

2. Allen VB, Gurusamy KS, Takwoingi Y, Kalia A, Davidson BR. Diagnostic accuracy of laparoscopy following computed tomography (CT) scanning for assessing the resectability with curative intent in pancreatic and periampullary cancer. Cochrane Database Syst Rev. 2016;7(7):CD009323.

3. Majlesara A, Golriz M, Hafezi M, Saffari A, Stenau E, Maier-Hein L, et al. Indocyanine green fluorescence imaging in hepatobiliary surgery. Photodiagn Photodyn Ther. 2017;17:208-15

4. Newton AD, Predina J, Mizelle J, Connolly C, Dunbar A, Baldassari M, et al. Intraoperative near-infrared imaging with second window Indocyanine green for pancreatic adenocarcinoma. J Am Coll Surg. 2017;225:S193.

5. Newton AD, Predina JD, Shin MH, Frenzel-Sulyok LG, Vollmer CM, Drebin JA, et al. Intraoperative near-infrared imaging can identify neoplasms and aid in real-time margin assessment during pancreatic resection. Ann Surg. 2019; 270:12-20.

6. Liberale G, Vankerckhove S, Caldon MG, Ahmed B, Moreau M, Nakadi IE, et al. Fluorescence imaging after indocyanine green injection for detection of peritoneal metastases in patients undergoing cytoreductive surgery for peritoneal carcinomatosis from colorectal cancer: a pilot study. Ann Surg. 2016;264:1110-5.

7. Handgraaf HJM, Sibinga Mulder BG, Shahbazi Feshtali S, Boogerd LSF, van der Valk MJM, Fariña Sarasqueta A, et al. Staging laparoscopy with ultrasound and near-infrared fluorescence imaging to detect occult metastases of pancreatic and periampullary cancer. PLoS One. 2018;13:e0205960.

\section{Publisher's Note}

Springer Nature remains neutral with regard to jurisdictional claims in published maps and institutional affiliations. 